\title{
INTERNAÇÕES POR ABUSO DE ÁLCOOL EM PRESIDENTE PRUDENTE NOS ÚLTIMOS TRÊS ANOS
}

Caroline Lopes Tamada', Maria Julia Santos Basana', Leonardo Santos de Souza², Camélia Santina Murgo ${ }^{1}$

Universidade do Oeste Paulista - UNOESTE, ${ }^{1}$ Faculdade de Medicina, ${ }^{2}$ Programa de Pós Graduação Mestrado em Educação, Presidente Prudente, SP. E-mail: carolinetamada@hotmail.com

\section{RESUMO}

Estudos destacam o alcoolismo como um sério problema para a saúde pública brasileira, assim como, compreende-se que intervenções eficientes diante deste cenário precisam levar em consideração o contexto específico e multidimensional deste público. Portanto, o objetivo deste estudo é descrever o perfil de internações devido ao abuso de álcool entre 2015 e 2017 na cidade de Presidente Prudente, por meio do portal da saúde DATASUS (TABNET). Trata-se de uma investigação transversal e descritiva, de caráter quantitativo. Foi possível identificar prevalência do sexo masculino em relação ao número de internações a nível regional e estadual. Entretanto, não foi possível estabelecer associações significativas entre períodos do ano de maior incidência de hospitalizações. Conclui-se que novas estratégias interinstitucionais e interdisciplinares precisam ser adotadas visando a erradicação da patologia a medida que promove saúde mental, especialmente nos homens.

Palavras-chave: Alcoolismo, Intoxicação Alcoólica, Hospitalização, Transtornos Mentais.

\section{HOSPITALIZATIONS FOR ALCOHOL ABUSE IN PRESIDENTE PRUDENTE IN THE LAST THREE YEARS}

\begin{abstract}
Studies highlight alcoholism as a serious problem for Brazilian public health, as well as, it is understood that efficient interventions in this scenario need to take into account the specific and multidimensional context of this public. Therefore, the objective of this study is to describe the profile of hospitalizations due to alcohol abuse between 2015 and 2017 in the city of Presidente Prudente, through the health portal DATASUS (TABNET). It is a cross-sectional and descriptive investigation, of a quantitative nature. It was possible to identify male prevalence in relation to the number of hospitalizations at regional and state level. However, it was not possible to establish significant associations between periods of the year with the highest incidence of hospitalizations. It is concluded that new interinstitutional and interdisciplinary strategies need to be adopted in order to eradicate the pathology, as it promotes mental health, especially in men.
\end{abstract}

Keywords: Alcoholism, Alcoholic Intoxication, Hospitalization, Mental Disorders. 


\section{INTRODUÇÃO}

A ingestão de álcool tem aumentado expressivamente, especialmente caracterizar-se como uma droga psicoativa lícita e de fácil acesso pela população, assim como, o consumo abusivo e dependente de tal substância, denominado como alcoolismo, também tem aumentado significativamente nos últimos anos.

O alcoolismo é uma doença mental de natureza multidimensional e complexa que atinge pessoas de todas as classes socais, considerado pela Organização Mundial da Saúde (OMS) como uma doença grave e como um problema de saúde pública, que pode levar a uma série de outras patologias. Entretanto, grande parte da população que por sua vez desconhece os malefícios do consumo de bebidas alcoólicas a médio e longo praz, como as doenças cardiovasculares, cirrose e complicações, bem como aumentar as chances de envolver-se em acidentes e conflitos sociais e familiares[1].

Ao ingerir o álcool, inicialmente, ocorre uma sensação de bem-estar, provocada pela atividade descontrolada de diversas áreas cerebrais. Entretanto, uso de etílicos leva posteriormente a um estado de "depressão", visto que o álcool é considerado um depressor do sistema nervoso central[2]. Dessa maneira, o álcool é uma droga que não acarreta em consequências negativas perceptíveis à curto prazo.

Além da compreensão neuropsicofisiológica e bioquímica do mecanismo de ação no alcoolismo, é preciso compreender os fatores sociais, culturais, econômicos e geográficos que possam estar envolvidos no favorecimento da ingestão patológica do álcool, para que as estratégias em saúde sejam cada vez mais eficazes e eficientes sobre o tratamento da doença[3].

Nesta direção, o presente estudo, teve como objetivo analisar as internações por transtornos mentais e comportamentais devido ao abuso de álcool, por sexo e ano de internação hospitalar. Verificou-se a possibilidade da existência de sazonalidade entre os anos analisados, estabelecendo comparativos entre os residentes no município de Presidente Prudente em relação ao estado de São Paulo.

\section{MÉTODO}

Trata-se de um estudo de caráter quantitativo, descritivo, com recorte transversal. Por tratar-se de uma investigação que faz uso apenas dados de origem secundária, dispensa a aprovação pelo Comitê de Ética em Pesquisa. Para a coleta dos dados, utilizou-se o portal da saúde DATASUS. Foi selecionado, em informações de saúde Epidemiológicas e Morbidade (TABNET), a opção Morbidade Hospitalar do SUS (SIH/SUS) do estado de São Paulo geral, por local de residência. A Lista de Morbidade do CID-10 escolhida foi a dos transtornos mentais e comportamentais devido uso de álcool, analisando-se o período de janeiro de 2015 a dezembro de 2017. Após coletar o número total de internações de em SP e no município de Presidente Prudente, os dados foram analisados por meio do Software Microsoft Excel e categorizados em tabelas organizativas.

\section{RESULTADOS}

Observou-se em todos os anos analisados a prevalência na internação de homens, tanto em Presidente Prudente, como no estado de São Paulo, visto que no referido município somados os três anos foram notificados 559 homens internados e 48 mulheres, e no estado de São Paulo 22053 homens e 2095 mulheres, conforme indicados nas tabelas 1 e 2. Na cidade de Presidente Prudente, no ano de 2015, o mês de maio obteve o maior número de internações ( $\mathrm{n}=38$ ), representando $12,8 \%$ do total. O mês com menor índice foi dezembro, apontando $4,1 \%$ do total de internações $(n=12)$. Ainda na cidade de Presidente Prudente, no que diz respeito à 2016, o mês de maior prevalência foi setembro, totalizando com 22 ocorrências (12\%). Em contrapartida, o mês com menor incidência, novamente foi o mês de dezembro com $9(4,9 \%)$ internações. Já no 
ano de 2017 o mês com maior número de internações foi janeiro, apontando 19 casos (15\%) e dezembro mais uma vez apresenta o menor índice, com 3,1\% ( $n=4)$, demonstrados na tabela 1.

No que tange ao estado de São Paulo em 2015 houve predomínio no mês de julho, totalizando 909 (9,1\%) internações e o menor índice em dezembro com 731 (7,3\%) ocorrências. 0 mês de prevalência em 2016, diferentemente do ano anterior analisado, foi o mês de janeiro, revelando $799(9,8 \%)$ internações. Entretanto, o menor índice novamente foi no mês de dezembro com 618 (7,6\%) casos. Em 2017 os meses com menor e maior incidência corroboram o ano de 2016 destacando janeiro como o mês de maior prevalência com 647 casos (9,5\%) e dezembro o período com o menor índice de internações, com 373 (5,5\%) internações, conforme explicitados na tabela 2. Não foi identificar uma relação significativa de um mês dentre os aos analisados e maior incidência de internações. Em contrapartida, tanto em Presidente Prudente quanto em São Paulo, o mês de dezembro apareceu em todos os anos analisados como o período de menor registro de internações.

No ano de 2015, o total de internações de residentes por transtornos mentais e comportamentais devido ao uso de álcool em Presidente Prudente foi igual a 296, enquanto no estado de São Paulo foi 9971. Em 2016, foram 184 e 8174, respectivamente. Ainda, em 2017, foi registrado 127 no referido município e 6813 internações na capital (vide Tabela 1 e 2).

Tabela 1. Internações de residentes na cidade de Presidente Prudente por transtornos mentais e comportamentais devido ao uso de álcool segundo sexo e mês/ano de atendimento:

\begin{tabular}{|c|c|c|c|c|c|c|}
\hline $\begin{array}{l}\text { Ano/mês de } \\
\text { atendimento }\end{array}$ & Masculino & $\mathrm{M} \%$ & Feminino & F\% & Total & $\%$ \\
\hline janeiro/2015 & 18 & $6,1 \%$ & 1 & $0,3 \%$ & 19 & $6,4 \%$ \\
\hline fevereiro/2015 & 13 & $4,4 \%$ & - & - & 13 & $4,4 \%$ \\
\hline março/2015 & 29 & $9,8 \%$ & 3 & $1,0 \%$ & 32 & $10,8 \%$ \\
\hline abril/2015 & 24 & $8,1 \%$ & 1 & $0,3 \%$ & 25 & $8,4 \%$ \\
\hline maio/2015 & 34 & $11,5 \%$ & 4 & $1,4 \%$ & 38 & $12,8 \%$ \\
\hline junho/2015 & 30 & $10,1 \%$ & 3 & $1,0 \%$ & 33 & $11,1 \%$ \\
\hline julho/2015 & 24 & $8,1 \%$ & - & - & 24 & $8,1 \%$ \\
\hline agosto/2015 & 28 & $9,5 \%$ & 1 & $0,3 \%$ & 29 & $9,8 \%$ \\
\hline setembro/2015 & 16 & $5,4 \%$ & 2 & $0,7 \%$ & 18 & $6,1 \%$ \\
\hline outubro/2015 & 23 & $7,8 \%$ & 1 & $0,3 \%$ & 24 & $8,1 \%$ \\
\hline novembro/2015 & 25 & $8,4 \%$ & 4 & $1,4 \%$ & 29 & $9,8 \%$ \\
\hline dezembro/2015 & 10 & $3,4 \%$ & 2 & $0,7 \%$ & 12 & $4,1 \%$ \\
\hline Total de 2015 & 274 & $92,6 \%$ & 22 & $7,4 \%$ & 296 & $100 \%$ \\
\hline janeiro/2016 & 17 & $9,2 \%$ & 3 & $1,6 \%$ & 20 & $10,9 \%$ \\
\hline fevereiro/2016 & 17 & $9,2 \%$ & 1 & $0,5 \%$ & 18 & $9,8 \%$ \\
\hline março/2016 & 14 & $7,6 \%$ & 3 & $1,6 \%$ & 17 & $9,2 \%$ \\
\hline
\end{tabular}




\begin{tabular}{|c|c|c|c|c|c|c|}
\hline abril/2016 & 10 & $5,4 \%$ & - & - & 10 & $5,4 \%$ \\
\hline maio/2016 & 10 & $5,4 \%$ & - & - & 10 & $5,4 \%$ \\
\hline junho/2016 & 14 & $7,6 \%$ & 3 & $1,6 \%$ & 17 & $9,2 \%$ \\
\hline julho/2016 & 17 & $9,2 \%$ & 2 & $1,1 \%$ & 19 & $10,3 \%$ \\
\hline agosto/2016 & 13 & $7,1 \%$ & 1 & $0,5 \%$ & 14 & $7,6 \%$ \\
\hline setembro/2016 & 20 & $10,9 \%$ & 2 & $1,1 \%$ & 22 & $12,0 \%$ \\
\hline outubro/2016 & 11 & $6,0 \%$ & 2 & $1,1 \%$ & 12 & $6,5 \%$ \\
\hline novembro/2016 & 15 & $8,2 \%$ & - & - & 15 & $8,2 \%$ \\
\hline dezembro/2016 & 8 & $4,3 \%$ & 1 & $0,5 \%$ & 9 & $4,9 \%$ \\
\hline Total de 2016 & 166 & $90,2 \%$ & 18 & $9,8 \%$ & 184 & $100 \%$ \\
\hline janeiro/2017 & 18 & $14,2 \%$ & 1 & $0,8 \%$ & 19 & $15,0 \%$ \\
\hline fevereiro/2017 & 13 & $10,2 \%$ & 2 & $1,6 \%$ & 15 & $11,8 \%$ \\
\hline março/2017 & 8 & $6,3 \%$ & 1 & $0,8 \%$ & 9 & $7,1 \%$ \\
\hline abril/2017 & 7 & $5,5 \%$ & - & - & 7 & $5,5 \%$ \\
\hline maio/2017 & 8 & $6,3 \%$ & 1 & $0,8 \%$ & 9 & $7,1 \%$ \\
\hline junho/2017 & 10 & $7,9 \%$ & - & - & 10 & $7,9 \%$ \\
\hline julho/2017 & 13 & $10,2 \%$ & 1 & $0,8 \%$ & 14 & $11,0 \%$ \\
\hline agosto/2017 & 15 & $11,8 \%$ & - & - & 15 & $11,8 \%$ \\
\hline setembro/2017 & 10 & $7,9 \%$ & - & - & 10 & $7,9 \%$ \\
\hline outubro/2017 & 8 & $6,3 \%$ & - & - & 8 & $6,3 \%$ \\
\hline novembro/2017 & 5 & $3,9 \%$ & 2 & $1,6 \%$ & 7 & $5,5 \%$ \\
\hline dezembro/2017 & 4 & $3,1 \%$ & - & - & 4 & $3,1 \%$ \\
\hline Total de 2017 & 119 & $93,7 \%$ & 8 & $6,3 \%$ & 127 & $100 \%$ \\
\hline
\end{tabular}

Fonte: Ministério da Saúde - Sistema de Informações Hospitalares do SUS (SIH/SUS) [4]. Nota: Dados trabalhados pelo autor

Tabela 2. Internações de residentes no estado de São Paulo por transtornos mentais e comportamentais devido ao uso de álcool segundo sexo e mês/ano de atendimento:

\begin{tabular}{lcccccc}
\hline $\begin{array}{l}\text { Ano/mês de } \\
\text { atendimento }\end{array}$ & Masculino & M\% & Feminino & F\% & Total & $\%$ \\
\hline janeiro/2015 & 788 & $7,9 \%$ & 99 & $1,0 \%$ & 887 & $8,9 \%$ \\
fevereiro/2015 & 690 & $6,9 \%$ & 81 & $0,8 \%$ & 771 & $7,7 \%$ \\
março/2015 & 786 & $7,9 \%$ & 95 & $1,0 \%$ & 881 & $8,8 \%$
\end{tabular}




\begin{tabular}{|c|c|c|c|c|c|c|}
\hline abril/2015 & 757 & $7,6 \%$ & 104 & $1,0 \%$ & 861 & $8,6 \%$ \\
\hline maio/2015 & 708 & $7,1 \%$ & 98 & $1,0 \%$ & 806 & $8,1 \%$ \\
\hline junho/2015 & 739 & $7,4 \%$ & 80 & $0,8 \%$ & 819 & $8,2 \%$ \\
\hline julho/2015 & 817 & $8,2 \%$ & 92 & $0,9 \%$ & 909 & $9,1 \%$ \\
\hline agosto/2015 & 802 & $8,0 \%$ & 97 & $1,0 \%$ & 899 & $9,0 \%$ \\
\hline setembro/2015 & 765 & $7,7 \%$ & 97 & $1,0 \%$ & 862 & $8,6 \%$ \\
\hline outubro/2015 & 683 & $7,9 \%$ & 103 & $1,0 \%$ & 786 & $7,9 \%$ \\
\hline novembro/2015 & 663 & $6,6 \%$ & 96 & $1,0 \%$ & 759 & $7,6 \%$ \\
\hline dezembro/2015 & 659 & $6,6 \%$ & 72 & $0,7 \%$ & 731 & $7,3 \%$ \\
\hline Total de 2015 & 8857 & $88,8 \%$ & 1114 & $11,2 \%$ & 9971 & $100 \%$ \\
\hline janeiro/2016 & 704 & $8,6 \%$ & 95 & $1,2 \%$ & 799 & $9,8 \%$ \\
\hline fevereiro/2016 & 598 & $7,3 \%$ & 75 & $0,9 \%$ & 673 & $8,2 \%$ \\
\hline março/2016 & 617 & $7,5 \%$ & 89 & $1,1 \%$ & 706 & $8,6 \%$ \\
\hline abril/2016 & 566 & $6,9 \%$ & 77 & $0,9 \%$ & 643 & $7,9 \%$ \\
\hline maio/2016 & 568 & $6,9 \%$ & 82 & $1,0 \%$ & 650 & $8,0 \%$ \\
\hline junho/2016 & 578 & $7,1 \%$ & 83 & $1,0 \%$ & 661 & $8,1 \%$ \\
\hline julho/2016 & 567 & $6,9 \%$ & 80 & $1,0 \%$ & 647 & $7,9 \%$ \\
\hline agosto/2016 & 668 & $8,2 \%$ & 100 & $1,2 \%$ & 768 & $9,4 \%$ \\
\hline setembro/2016 & 613 & $7,5 \%$ & 67 & $0,8 \%$ & 680 & $8,3 \%$ \\
\hline outubro/2016 & 597 & $7,3 \%$ & 87 & $1,1 \%$ & 684 & $8,4 \%$ \\
\hline novembro/2016 & 570 & $7,0 \%$ & 75 & $0,9 \%$ & 645 & $7,9 \%$ \\
\hline dezembro/2016 & 542 & $6,6 \%$ & 76 & $0,9 \%$ & 618 & $7,6 \%$ \\
\hline Total de 2016 & 7188 & $87,9 \%$ & 986 & $12,1 \%$ & 8174 & $100 \%$ \\
\hline janeiro/2017 & 567 & $8,3 \%$ & 80 & $1,2 \%$ & 647 & $9,5 \%$ \\
\hline fevereiro/2017 & 493 & $7,2 \%$ & 68 & $1,0 \%$ & 561 & $8,2 \%$ \\
\hline março/2017 & 536 & $7,9 \%$ & 79 & $1,2 \%$ & 615 & $9,0 \%$ \\
\hline abril/2017 & 488 & $7,2 \%$ & 77 & $1,1 \%$ & 565 & $8,3 \%$ \\
\hline maio/2017 & 503 & $7,4 \%$ & 73 & $1,1 \%$ & 576 & $8,5 \%$ \\
\hline junho/2017 & 569 & $8,4 \%$ & 71 & $1,0 \%$ & 640 & $9,4 \%$ \\
\hline julho/2017 & 480 & $7,0 \%$ & 64 & $0,9 \%$ & 544 & $8,0 \%$ \\
\hline agosto/2017 & 554 & $8,1 \%$ & 63 & $0,9 \%$ & 617 & $9,1 \%$ \\
\hline
\end{tabular}




$\begin{array}{lcccccc}\text { setembro/2017 } & 521 & 7,6 \% & 56 & 0,8 \% & 577 & 8,5 \% \\ \text { outubro/2017 } & 502 & 7,4 \% & 75 & 1,1 \% & 577 & 8,5 \% \\ \text { novembro/2017 } & 459 & 6,7 \% & 62 & 0,9 \% & 521 & 7,6 \% \\ \text { dezembro/2017 } & 336 & 4,9 \% & 37 & 0,5 \% & 373 & 5,5 \% \\ \text { Total de } 2017 & \mathbf{6 0 0 8} & \mathbf{8 8 , 2 \%} & \mathbf{8 0 5} & \mathbf{1 1 , 8 \%} & \mathbf{6 8 1 3} & \mathbf{1 0 0 \%}\end{array}$

Fonte: Ministério da Saúde - Sistema de Informações Hospitalares do SUS (SIH/SUS) [4].

Nota: Dados trabalhados pelo autor.

\section{DISCUSSÃO}

É possível afirmar que houve uma tendência ao sexo masculino, pois esse é o grupo que mais utiliza de bebidas alcoólicas e/ou outros tipos de substâncias químicas[5]. O fato de que o número de internações em Presidente Prudente e São Paulo terem diminuído nos últimos 3 anos pode ser um indicativo de que população esteja mais conscientizada, ingerindo álcool com moderação. Entretanto, o alcoolismo ainda é considerado um sério problema de saúde pública. 0 consumo excessivo dessa droga pode causar diversos problemas físicos, mentais e comportamentais. Ademais, o abuso de bebidas alcoólicas é uma causa para acidentes e internações nos hospitais do país [6]

Frente ao exposto, conclui-se que em Presidente Prudente nos três anos avaliados, o número de internações de residentes por transtornos mentais e comportamentais devido ao uso de álcool diminuiu. O fato das taxas terem reduzido também foi observado no estado de São Paulo. É prevalente a internação de homens, e não foi encontrada relação expressiva referente à mês e número de internações. Sugerem-se pesquisas com enfoque na busca por relações de dependência por meio de análises estatísticas mais robustas, abrangendo uma amostra de casos ampla para que generalizações possam ser estabelecidas com maior segurança, assim como, investigações multicêntricas possam correlacionar perfis psicossociológicos de homens e mulheres em relação ao consumo abusivo de álcool para que estratégias interdisciplinares e interinstitucionais possam ser mais efetivas frente ao comportamento de risco à saúde da pessoa alcoolista.

\section{CONFLITO DE INTERESSES}

Os autores declaram não haver qualquer potencial conflito de interesse que possa interferir na imparcialidade deste trabalho científico.

\section{REFERÊNCIAS}

1- MEDEIROS, ME. Alcoolismo: uma breve revisão - 2018 - Disponível em: <http://www.psicologia.pt/artigos/textos/A1174.pdf>

2- PAULIN, LFRS. Conceito, etiologia e diagnostico do alcoolismo: uma revisão. Revista de ciências médicas - PUCCAMP, Campinas, 3(1), p 5-8, janeiro/abril, 1994. Disponível em: <https://seer.sis.puccampinas.edu.br/seer/index.php/cienciasmedicas/article/view/1423/1397>

3- DIEHL, A.; CRUZ CORDEIRO, D.; LARANJEIRA, R. Dependência química: prevenção, tratamento e políticas públicas. Porto Alegre: Artmed, 2011.

4- $\quad$ Ministério da Saúde - Sistema de Informações Hospitalares do SUS (SIH/SUS) 
5- CAMPANA, AAM; ZALESKI, M.; RAMOS, SP. Abuso e Dependência de Álcool. [S.I.]: Associação Brasileira de Psiquiatria Sociedade Brasileira de Medicina da Família e Comunidade, [31 de março de 2012]. 36 p. Disponível em: <http://www.sbmfc.org.br/media/file/diretrizes/02abuso e dependenia de alcool.pdf>

6- DE ANDRADE, Arthur Guerra; ANTHONY, James C. Álcool e suas conseqüências: uma abordagem multiconceitual. Câmara Brasileira do Livro, SP, Brasil: Editora Manole Ltda., 2009. 16 p. 